\title{
The Hardware Design of Intelligent Management System for Traffic
}

\author{
Jinfang He, *Ling Long \\ College of Electrical \& Information Engineering, \\ Southwest University for Nationalities, \\ Chengdu, 610041, China
}

\begin{abstract}
In this paper, we put forward a new type of intelligent assistant management system, and implement the hardware design of intelligent assistant system for pedestrian traffic in city. This system uses MCU as main control chip, also include san other four parts: mechanical arm, detector, image acquisition and voice prompt, all parts constitute an assistant management system together. The main control module receives and processes the information returned by sensors, sends instructions to mechanical arm. Then system can achieve a good traffic order intelligently and efficiently.
\end{abstract}

Keywords-urban intersection; pedestrian traffic; intelligence management; mechanical arm.

\section{INTRODUCTION}

With the rapid development of economic construction and urbanization, traffic capacity cannot meet the needs of continuously growing vehicles which makes the traffic in city a prominent problem. Since congestion and traffic accidents append frequently, the traffic is getting tenser and tenser [1]. So the intelligent transportation system comes into being. Intelligent Transportation System is a real-time, accurate, effective and inter-graded transportation management system, which can be used on a large scale and in many fields, its aim is to apply advanced information technology, data transmission technology, electronic sensor technology, control technology and computer technology to the ground traffic management system. This system direct traffic through the mechanical arm which simulates actions of traffic police, it monitors the intersections through detectors and give out voice prompt and warning.

\section{DESIGN OF SYSTEM HARDWARE STRUCTURE}

The system can be divided into five modules: MCU main control module, mechanical automatic control module, detect module, image acquisition module and voice prompt module.MCU main control module, which is the data processing and instructions controlling central of the whole system, receives the feedback data of each subsystem to conduct comprehensive treatment and judgment, and sent control instructions to control other modules. According to the data and instructions sent by MCU main control module, Mechanical automatic control module controls and conducts mechanical arm to finish each command gesture. Detect module monitors intersection in real-time, to check if there is someone running the red light, and sends feedback information to MCU main control module promptly. Image acquisition module gathers and deals with image data which synchronously displayed by TFTLCD .Voice prompt module sends voice warning without delay when the behavior of infringement happens.

\section{A. MCU main control module}

We adopt 32-bits Kinetics micro-controller, MK60DN512ZVMD10, as MCU main controller.MCU main controller collects to every module in hardware. MCU would receive feedback information from other module. MCU deals with information and send instructions to control other module, to make them work in order. Kinetics series micro-controller is produced by free scale co. in the second half year of 2010, it is the first micro-controller based on the kernel of ARM CortexM4.K60 minimum system consists of MK60DN512ZVLQ10,power module, I/O port Module,8 ways of PWM output interface [2]. I/O ports are used to control and exchange information with other module, external keys and liquid crystal display module are for debugging, 8 ways of PWM output interface output the PWM wave generated from MK60DN512ZVLQ10 which controls the steering engine [3-4].Power module supply power to the system.AMS1117 stabilizes the voltage in 3.3V to supply power to chips.LM2596-ADJ can generate adjustable voltage through adjustable resistor, so that it can provide different voltage to different devices.

\section{B. Mechanical automatic control module}

We use steering engine to achieve the function of the mechanical arm, the function of arm is to achieve traffic controlling actions, so there's no need to consider whether it can bear heavy things, thus the actuator which uses steering engine as arm is feasible. Steering engine has three input wires, red one in the middle is power wire, the black one is ground wire, and another is signal controlling wire. The control signal is pulse width modulation(PWM) signal whose period is $20 \mathrm{~ms}$, the pulse width changes linearly from $0.5 \mathrm{~ms}$ to $2.5 \mathrm{~ms}$, which corresponds to the superimposition during 0 to 180 angles. That is to say, if we give it a certain pulse width, its output shaft will remain in a corresponding angle, no matter how the external torque changes, it will not change the output angle to the new corresponding position until we give it another signal.

The steering engine has an internal reference circuit, which generates a reference signal with $20 \mathrm{~ms}$ of period and $1.5 \mathrm{~ms}$ of width, and there is a comparator, which compares external signal with the reference signal to judge the direction and magnitude, so it can produce the 
rotate signal for the motor. A mechanical arm consists of four steering engines, four metal helms, four long U-shape brackets made in hard aluminum alloy, two L-shape brackets made in hard aluminum alloy, three U-shape beams made in hard aluminum and screws. U-shape beams formed the platform to fix the arm. Four U-shape brackets are used as branches. Two steering engines and two helms functioned as joint, separately control the movements of two directions: up and down, left and right. By controlling matches of four steering engines rotation angles, the mechanical arm can realize the actions that a traffic police does.

\section{Detection module}

Detection module can be divided into the body infrared detection and the ultrasonic detection [5]. The physical picture of body infrared detector is shown in fig.1, the body infrared detector is the inductor to human body, the body infrared detection can identify whether there is a pedestrian in the detection area. Ultrasonic detection can determine if there is a red light behavior in the detection area according to the detection range.

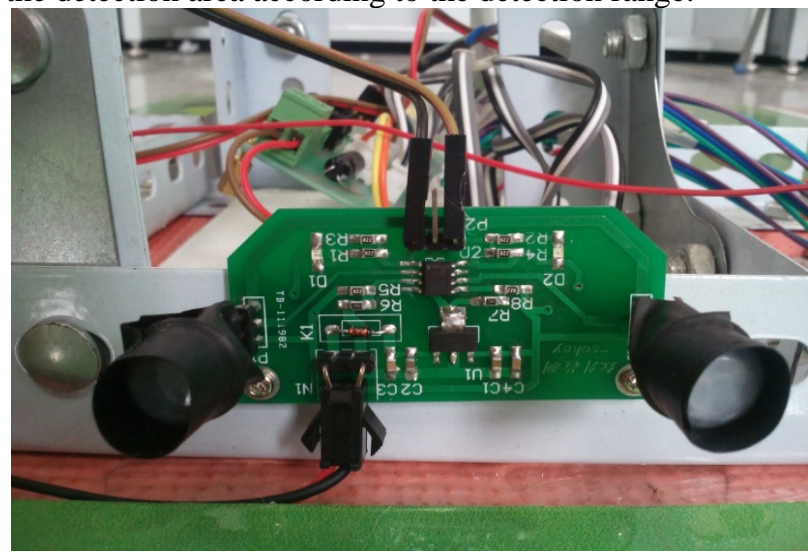

Fig.1. Physical picture of body infrared detector

The body infrared detection adopts SC0322 human body sensor for detecting.SC0322 human body sensor is a digital intelligent automatic controlling product, which based on the technology of passive body infrared. The external circuit of the product is very simple. In the external detection test, it has high sensitivity and reliability. Therefore, it's widely used in all kinds of automatic induction detection circuits.

SC0322 body sensors have stronger anti-jamming ability, whose internal uses digital signal processing and outputs high and low level directly. When the input voltage is $\mathrm{DC} 5 \mathrm{~V}$, the output voltage is DC2.3V. It can be triggered repeatedly, after outputting high level ,during the time-delay period, if there is a human in its sensing area, its output will not change until the people leave, time-delay comes again when people leave then it goes down to low level. Induction module will be automatically postponed to a delaying time after detecting any activity of human body, and making the last activity time as the beginning of delaying time. System main controller can monitor crowd movements by monitoring the pins of body sensor.

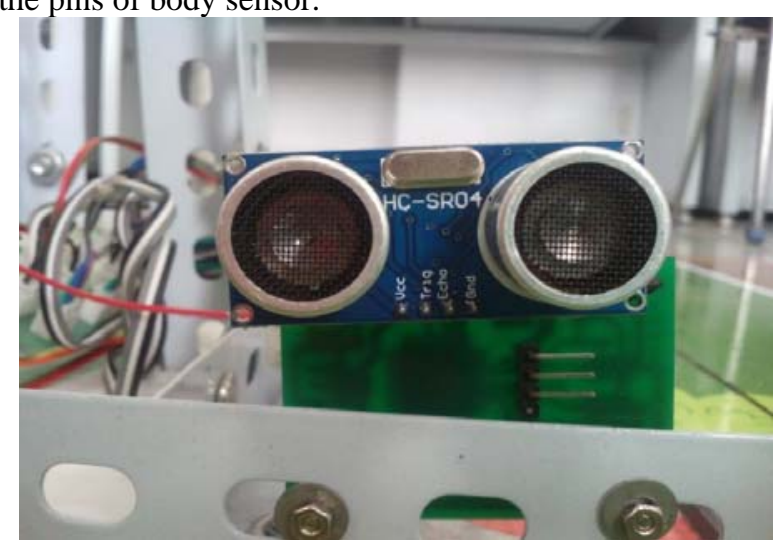

Fig.2. Physical picture of ultrasonic detector

Ultrasonic detection part adopts HC-SR04 ultrasonic range finder for detection[5], physical picture of ultrasonic detector is shown in fig.2. According to the mobile measuring characteristics of HC-SR04 ultrasonic range finder, we use it in the dynamic test to detect if anyone go through the red light. It's simple to use HCSR04, the only thing we need to do is to send a high level above 10us,and ultrasonic launcher will automatically sent eight square waves in $40 \mathrm{KHz}$. When faced with obstacles, receiving device automatically detects if there is a returned signal. If the signal returns, it can wait for the output of high level in the output port. By dealing with returned information of HC-SR04 ultrasonic range finder, system main controller can judge if anyone go through the red light, so as to realize detection.

\section{Road voice prompt module}

The road voice prompt module makes the prompt according to the traffic lights. As is shown fig. 3, the road voice prompt system consists of SYN6288 voice chips, STC89C52 single chip microcomputer [6], LM2940 voltage regulator chips, external audio interface and the main control module connection interface and so on.

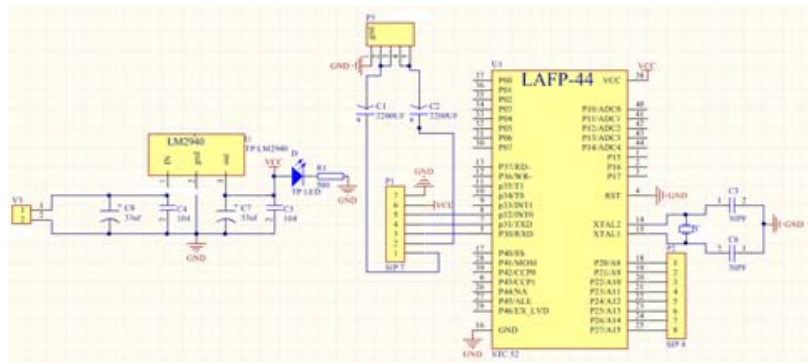

Fig.3. Schematic diagram of voice prompt module

As we can see in fig. 3, the power supplies to chips through the voltage stabilizing circuit. Even if the input voltage fluctuates fiercely, the voltage that stabilized by LM2940 can offer MCU and voice chip a stable working voltage, in this way, the whole system can work in order. LED is the Working indicator light for the power and 
chips.U1 is STC89C51,its P3.0/RXD 、 P3.1/TXD pins connect to TXD,RXD pins of SYN6288,aimed to send the voice chips the text data which is to be synthesized. Port P2 exchanges information with the outside, its low 4-bits receive controlling instructions, high 4-bits return voice prompt system working state to outside [7].The signal of traffic lights, whether a prompt should be given, what kind of prompt should be given, and all kinds of information and instructions can be gained from port P2.Audio interface output the electrical signals prompt voice transformed from the text by SYN6288,then,put the signal to audio power amplifier and speaker, in this method, the prompt voice could be heard.

\section{CONCLUSIONS}

The system combines mechanical automatic control, detection, image acquisition and road voice prompt in one, and it works in order under the coordination of MCU main controller. When the MCU main controller receives the warning of cars running the red light, voice prompt module, mechanical automatic control module, detecting module and image acquisition module immediately react according to the instructions given by the MCU main controller. The voice prompt module sends voice warning; the mechanical automatic module makes guidance move to dissuade; and the image acquisition module collects the images of breaking rules behaviors. All the modules work together around the MCU to realize the function of intelligent assistant management system for pedestrian traffic in city in assistant directing.

\section{ACKNOWLEDGEMENTS}

This work was financially supported by College Students' innovation and entrepreneurship training plan of Southwest University for Nationalities NO.S201410656018.

\section{REFERENCES}

[1] Jin Mao-Qing. Development of Intelligent Transportation in China. Transportation Science and Technology,2,pp. 140-142, 2013.

[2] Zhang Yi-Kun, Chen Shan-Jiu and Qiu Xue-Hong. The Principle and Application of Single Chip Microcomputer, Xi'an: University of Electronic Science and Technology Press,1998.

[3] Guo Tian-Xiang. New Conception tutorial of $51 \mathrm{MCU}$ in C language. Beijing: Electronic Industry Press,2009.

[4] Tan Hao-Qiang. C Program Design. Beijing: Qinghua University Press,2005.

[5] Meng Li-Fan, Lan Jin-Hui: Principle and Application of Sensor. Beijing: Electronic Industry Press, 2011.

[6] Cheng Qin and Hou Wei. Household Integrated Circuit with the Control and Detection. Beijing: China Metrology Press, 2005.

[7] Luo Wei-Ping and Li De-Jun: Fundamentals and Application of MCU. Wuhan: Huazhong University of Science and Technology Press, 2012. 